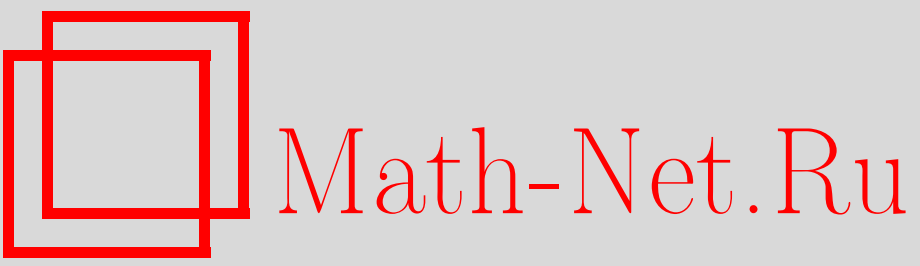

W. Ham, K. Zhou, A short note on the volume of hypersphere, Теория вероятн. и ее примен., 2009, том 54, выпуск 4, 809-814

DOI: https://doi.org/10.4213/tvp3596

Использование Общероссийского математического портала Math-Net.Ru подразумевает, что вы прочитали и согласны с пользовательским соглашением

http://www.mathnet.ru/rus/agreement

Параметры загрузки:

IP : 34.229 .108 .108

26 апреля 2023 г., 15:55:53 
4. Talagrand $M$. The Glivenko-Cantelli problem, ten years later. - J. Theoret. Probab., 1996 , v. 9, № 2, p. 371-384.

5. Nobel A., Dembo A. A note on uniform laws of averages for dependent processes. Statist. Probab. Lett., 1993, v. 17, № 3, p. 169-172.

6. Peligrad M. A note on the uniform laws for dependent processes via coupling. - J. Theoret. Probab., 2001, v. 14, № 4, p. 979-988.

7. Pollard D. Convergence of Stochastic Processes. New York: Springer, 1984, 215 p.

8. Биллингсли П. Сходимость вероятностных мер. М.: Наука, 1977, 351 с.

9. Bryc W. On the approximation theorem of I. Berkes and W. Philipp. - Demonstratio Math., 1982, v. 15, № 3, p. 807-816.

10. Ширяев А.Н. Вероятность, т. 2. М.: МЦНМО, 2004, 408 с.

11. Харди Г. Г., Литтльвуд Дж. Е., Полиа Г. Неравенства. М.: ИЛ, 1948, 456 с.

Поступила в редакцию 30.IX. 2008

(C) $2009 \mathrm{\Gamma}$

HAM W.*, ZHOU $\mathrm{K}^{* * *}$

\title{
A SHORT NOTE ON THE VOLUME OF HYPERSPHERE ${ }^{1}$
}

\begin{abstract}
Предлагается новый метод вычисления объема гиперсферы, использующий теорию вероятностей. Явное выражение для многократной свертки плотностей вероятности, которое следовало бы использовать, очень сложно. Но в нашем методе требуется не все явное выражение, а лишь часть информации, что позволяет вывести общую формулу для объема гиперсферы.
\end{abstract}

Ключевые слова и фразы: гиперсфера, вероятность, свертка, парадокс.

1. Introduction. In this note, a new approach to derive the volume of hypersphere is proposed. Even though the volume formula has been already derived and introduced in many books (see [1]-[3]), the method proposed in this note may be useful for solving many unsolved problems in hyperdimensional space. The proposed method is motivated from the probability theory, especially the mathematical fact that the probability density function of summation of two independent random variables can be expressed as a convolution of the two probability density functions. We would expect that the explicit expression of the multiple times convolution of the same probability density functions may converge to a Gaussian distribution regardless of its initial shape of probability density function. Also it is very complicated to describe the whole process of this multiple times convolution and to derive the explicit expression of the finite multiple times convolution. Fortunately, only a small part of information, such as values of the probability functions in the domain between 0 and 1 , is needed in the derivation of volume of hypersphere and this fact makes it possible to derive the general formula for the hypersphere. We also briefly review two conventional methods to derive the volume of hyperspere. Instinctive geometric properties of hypersphere is assumed and the gamma integral is used in the conventional approach

* Electronics and Information Division, Chonbuk National University, Chonju-si, Chonbuk, South Korea; e-mail: wcham@moak.chonbuk.ac.kr; this work was completed while the author was a visiting professor at Louisiana State University.

** Department of Electrical and Computer Engineering, Louisiana State University, Baton Rouge, LA 70803, USA; e-mail: kemin@ece.lsu.edu

1) This work was completed with the partial support of post BK21 project of Chonbuk National University. 
and these methods may be more concise compared with the new method we propose but instinctive geometric properties are not needed in our approach and this is the advantage of our approach.

2. Conventional method. In this section, we briefly review the conventional methods for deriving the formula of the volume of hypershpere.

2.1. Method A. At first, we review the method introduced by Hamming [3]. He used the following well-known property of gamma integral:

$$
\left[\Gamma\left(\frac{1}{2}\right)\right]^{n}=\pi^{n / 2}=\int_{-\infty}^{\infty} \cdots \int_{-\infty}^{\infty} e^{-r^{2}} d x_{1} \cdots d x_{n}
$$

where $r^{2}=x_{1}^{2}+\cdots+x_{n}^{2}$. He also assumed that the volume $V_{n}(r)$ of an $n$-dimensional sphere depends on the radius by reflection on the classical Euclidean geometry as follows:

$$
V_{n}(r)=C_{n} r^{n}
$$

He proposed the following equation by using the geometric intuition:

$$
\int_{-\infty}^{\infty} \cdots \int_{-\infty}^{\infty} e^{-r^{2}} d x_{1} \cdots d x_{n}=\int_{0}^{\infty} e^{-r^{2}} \frac{d V_{n}(r)}{d r} d r
$$

From the above equations, we have

$$
C_{n}=\frac{\pi^{n / 2}}{\Gamma(n / 2+1)}
$$

2.2. Method B. Further, we review the method introduced in [1]. Here, $S^{n}$ denotes the hypersurface of $n$-dimensional hypersphere. The volume contained inside $S^{n}$ is given by the integral

$$
V_{n}(r)=\int_{\sum_{i=1}^{n} x_{i}^{2} \leqslant r^{2}} d x_{1} \cdots d x_{n} .
$$

Going to polar coordinates $\left(r^{2}=\sum_{i=1}^{n} x_{i}^{2}\right)$ this becomes

$$
V_{n}(r)=\int_{S^{n}} d \Omega \int_{0}^{r} \lambda^{n-1} d \lambda .
$$

The first integral is the integral over all solid angles subtended by the sphere and is equal to its area $A(n)=2 \pi^{n / 2} / \Gamma(n / 2)$. The second integral is elementary and evaluates to $\int_{0}^{r} \lambda^{n-1} d \lambda=r^{n} / n$. Finally, the volume is

$$
V_{n}(r)=\frac{\pi^{n / 2}}{(n / 2) \Gamma(n / 2)} r^{n}=\frac{\pi^{n / 2}}{\Gamma(n / 2+1)} r^{n}
$$

Note that this formula works for $n \geqslant 0$. The first few cases are:

1) $n=2: \Gamma(2)=1$, hence $V_{2}(1)=\pi$ (this is the familiar result for the area of the unit circle);

2) $n=3: \Gamma(5 / 2)=3 \sqrt{\pi} / 4$, hence $V_{3}(1)=4 \pi / 3$ (this is the familiar result for the volume of the unit sphere);

3) $n=4: \Gamma(3)=2$, hence $V_{4}(1)=\pi^{2} / 2$.

3. Mathematical preliminary. We briefly introduce the convolution theory in random variables which will be used in the next section, see [4].

Theorem 3.1. The probability density function $p_{z}(z)$ of the summation of several independent random variables, such as $z=x_{1}+\cdots+x_{n}$, is expressed as follows:

$$
p_{z}(z)=p_{x_{1}}(z) * \cdots * p_{x_{n}}(z),
$$

where operator $*$ denotes the convolution of functions. 
Theorem 3.2. Let the random variable $x$ be uniformly distributed between -1 and 1 . Then, the probability density function of the random variable $x^{2}, p_{x^{2}}(z)$, is described by

$$
p_{x^{2}}(z)= \begin{cases}0 & \text { if } z<0 \\ (1 / 2) z^{-1 / 2} & \text { if } 0 \leqslant z \leqslant 1 \\ 0 & \text { otherwise. }\end{cases}
$$

Let us define the following probability density functions:

$$
\begin{gathered}
p_{1}(z)=p_{x^{2}}(z), \quad p_{2}(z)=p_{1}(z) * p_{1}(z), \quad \ldots, \\
p_{n}(z)=p_{1}(z) * \cdots * p_{1}(z)(n \text { times }) .
\end{gathered}
$$

The complete explicit expression of the above probability density function may converge to some types of Gaussian distribution function. Therefore, it is a very difficult problem to find the explicit forms of above probability density functions for finite $n$. But only the first part of information, i.e., the explicit form of above probability density functions in the domain of $z$ between 0 and 1 , is needed in the derivation of the volume of hypersphere. Because of this fact, we will only derive the explicit form of each probability function in the domain of $z$ between 0 and 1 in the next section.

4. Probability density functions. In this section, we want to derive the explicit form in the domain of $z$ between 0 and 1 for each probability function $p_{n}(z)$ defined in the previous section. From the mathematical preliminary, the probability density function $p_{2}(z)=p_{1}(z) * p_{1}(z)$ is expressed as follows:

$$
p_{2}(z)= \begin{cases}\int_{0}^{z} \frac{1}{4} \lambda^{-1 / 2}(z-\lambda)^{-1 / 2} d \lambda & \text { if } 0 \leqslant z \leqslant 1 \\ \int_{z-1}^{1} \frac{1}{4} \lambda^{-1 / 2}(z-\lambda)^{-1 / 2} d \lambda & \text { if } 1 \leqslant z \leqslant 2 \\ 0 & \text { otherwise. }\end{cases}
$$

By using substitution $\lambda=\tau^{2}$, we obtain $d \lambda=2 \tau d \tau$ and

$$
p_{2}(z)= \begin{cases}\int_{0}^{\sqrt{z}} \frac{1}{2}\left(z-\tau^{2}\right)^{-1 / 2} d \tau & \text { if } 0 \leqslant z \leqslant 1 \\ \int_{\sqrt{z-1}}^{1} \frac{1}{2}\left(z-\tau^{2}\right)^{-1 / 2} d \tau & \text { if } 1 \leqslant z \leqslant 2 \\ 0 & \text { otherwise. }\end{cases}
$$

By using substitution $\tau=\sqrt{z} \sin \theta$, we obtain

$$
p_{2}(z)= \begin{cases}\frac{\pi}{4} & \text { if } 0 \leqslant z \leqslant 1 \\ \frac{1}{2} \sin ^{-1}\left(\frac{1-z / 2}{z / 2}\right) & \text { if } 1 \leqslant z \leqslant 2 \\ 0 & \text { otherwise. }\end{cases}
$$

Now, we derive the first part, that is, in the domain of $z$ between 0 and 1 , of the probability density function $p_{3}(z)$ by using convolution of $p_{2}(z)$ and $p_{1}(z)$. It is easy to see that the first part of $p_{3}(z)$ can be expressed as follows:

$$
p_{3}(z)= \begin{cases}0 & \text { if } z<0 \\ \frac{\pi}{8} \int_{0}^{z}(z-\lambda)^{-1 / 2} d \lambda & \text { if } 0 \leqslant z \leqslant 1 \\ \text { not needed } & \text { otherwise }\end{cases}
$$


It is easy to obtain the following simplified equation for the first part of $p_{3}(z)$ :

$$
p_{3}(z)= \begin{cases}0 & \text { if } z<0 \\ \frac{\pi}{4} z^{1 / 2} & \text { if } 0 \leqslant z \leqslant 1 \\ \text { not needed } & \text { otherwise. }\end{cases}
$$

The important thing to note in derivation of the above equations is that we only need and use the function shape of $p_{1}$ and $p_{2}$ in the domain of $z$ between 0 and 1 to derive the explicit expression of function shape of $p_{3}$ in the domain of $z$ between 0 and 1 . The function shape of $p_{1}$ and $p_{2}$ in the domain of $z$ between 0 and 1 is so simple and this fact makes it possible to derive the explicit formula for $p_{3}$ in the domain of $z$ between 0 and 1 . In the similar way, we do not need to derive the explicit formula of $p_{n}(z)$ for $n=3,4, \ldots$ in the domain of $z$ beyond 1 and this fact makes it possible to derive explicit formula of the volume of the hypersphere. Now we can derive the general form of the first part, that is, in the domain of $z$ between 0 and 1 , of the probability density function $p_{n}(z)$ when $n$ is even, such as $n=2 m$, by using the multiple times convolution of $p_{2}(z)$ as follows:

$$
p_{2 m}(z)= \begin{cases}0 & \text { if } z<0 \\ p_{2}(z) * \cdots * p_{2}(z)(m \text { times }) & \text { if } 0 \leqslant z \leqslant 1 \\ \text { not needed } & \text { otherwise }\end{cases}
$$

It is easy to obtain the following simplified equation for the first part of $p_{2 m+2}(z)$ when $m \geqslant 1$ :

$$
p_{2 m+2}(z)= \begin{cases}0 & \text { if } z<0 \\ \left(\frac{\pi}{4}\right)^{m+1} \frac{z^{m}}{m !} & \text { if } 0 \leqslant z \leqslant 1 \\ \text { not needed } & \text { otherwise. }\end{cases}
$$

Further, we derive the general form of the first part of the probability density function when $n$ is odd, that is, when $n=2 m+2+1$, by using convolution of $p_{2 m+2}(z)$ and $p_{1}(z)$ as follows:

$$
p_{2 m+2+1}(z)= \begin{cases}0 & \text { if } z<0, \\ \int_{0}^{z}\left(\frac{\pi}{4}\right)^{(m+1)} \frac{1}{2} \lambda^{-1 / 2} \frac{(z-\lambda)^{m}}{m !} d \lambda & \text { if } 0 \leqslant z \leqslant 1, \\ \text { not needed } & \text { otherwise. }\end{cases}
$$

By using several partial integrations, we can obtain the following simplified form for $p_{2 m+2+1}(z)$ :

$$
p_{2 m+2+1}(z)= \begin{cases}0 & \text { if } z<0, \\ \left(\frac{\pi}{4}\right)^{(m+1)} \cdot \frac{2}{3} \cdot \frac{2}{5} \cdot \frac{2}{7} \cdots \frac{2}{2 m+1} z^{(2 m+1) / 2} & \text { if } 0 \leqslant z \leqslant 1, \\ \text { not needed } & \text { otherwise, }\end{cases}
$$

where $m \geqslant 1$. Here, we use the following equations which we obtain by using partial integration:

$$
\begin{aligned}
\int_{0}^{z}\left(\frac{\pi}{4}\right)^{m+1} & \cdot \frac{1}{2} \lambda^{-1 / 2} \frac{(z-\lambda)^{m}}{m !} d \lambda=\int_{0}^{z}\left(\frac{\pi}{4}\right)^{m+1} \cdot \frac{1}{2} \cdot 2 \cdot \lambda^{1 / 2} \frac{(z-\lambda)^{m-1}}{(m-1) !} d \lambda \\
& =\int_{0}^{z}\left(\frac{\pi}{4}\right)^{m+1} \cdot \frac{2}{3} \lambda^{3 / 2} \frac{(z-\lambda)^{m-2}}{(m-2) !} d \lambda \\
& =\int_{0}^{z}\left(\frac{\pi}{4}\right)^{(m+1)} \cdot \frac{2}{3} \cdot \frac{2}{5} \lambda^{5 / 2} \frac{(z-\lambda)^{m-3}}{(m-3) !} d \lambda=\cdots
\end{aligned}
$$


5. Volume formula of hypersphere. In this section, we propose a new method to derive the volume of $n$-dimensional unit hypersphere. Consider the $n$-dimensional hypercube and assume that the length of each edge of this hypercube is 2 . Let us think about choosing a point in this hypercube. Now we want to calculate the probability $p_{\text {hyper }}$ that the point chosen as above belongs to the $n$-dimensional unit hypersphere contained in the above hypercube. We can easily see that the probability $p_{\text {hyper }}$ is the ratio between the hypervolume $V_{s n}$ of unit hypersphere and hypervolume $V_{c n}$ of the hypercube defined above and it can be expressed as follows:

$$
\begin{aligned}
p_{\text {hyper }}= & \frac{\text { Volume of } V_{s n}}{\text { Volume of } V_{c n}} \\
& =\frac{\text { Volume of }\left\{\left(x_{1}, \ldots, x_{n}\right) \in \mathbf{R}^{n}: x_{1}^{2}+\cdots+x_{n}^{2}<1\right\}}{\text { Volume of }\left\{\left(x_{1}, \ldots, x_{n}\right) \in \mathbf{R}^{n}:-1 \leqslant x_{1} \leqslant 1, \ldots,-1 \leqslant x_{n} \leqslant 1\right\}},
\end{aligned}
$$

where $V_{c n}=2^{n}$. Now, we propose a way to calculate the volume of a hypersphere.

Theorem 5.1. The volume of $n$-dimensional hypersphere $V_{\text {sn }}$ is expressed as

$$
V_{s n}=C_{n} r^{n},
$$

where $r$ denotes the radius of hypersphere and $C_{n}$ denotes the volume of a unit hypersphere,

$$
C_{n}=2^{n} \cdot p_{\text {hyper }}, \quad p_{\text {hyper }}=\int_{0}^{1} p_{n}(z) d z .
$$

It is clear from the above theorem that we only need to integrate the probability density function in the domain of $z$ between 0 and 1 . From the property of convolution, we only need the information of probability density functions in the domain of $z$ between 0 and 1 to derive the same part of information of the convolution. It is clear that

$$
C_{n}= \begin{cases}\frac{\pi^{n / 2}}{(n / 2) !} & \text { if } n \text { is even, } \\ \frac{\pi^{(n-1) / 2} 2^{(n+1) / 2}}{3 \cdot 5 \cdot 7 \cdots n} & \text { if } n \text { is odd, }\end{cases}
$$

where $n \geqslant 2$. Therefore, we finally obtain the general form of volume for hypersphere as follows:

$$
V_{n}(r)= \begin{cases}\frac{\pi^{n / 2}}{(n / 2) !} r^{n} & \text { if } n \text { is even, } \\ \frac{\pi^{(n-1) / 2} 2^{(n+1) / 2}}{3 \cdot 5 \cdot 7 \cdots n} r^{n} & \text { if } n \text { is odd, }\end{cases}
$$

where $n \geqslant 2$. Therefore, if we use the gamma function the volume of $n$-dimensional hypersphere is expressed as

$$
V_{n}(r)=\frac{\pi^{n / 2}}{\Gamma(n / 2+1)} r^{n}
$$

The above formula is the same as conventional one introduced in the previous section.

6. Conclusion. In this short note, a new method to derive the volume of hypersphere is proposed and the approach is very different from conventional geometric approach. In this approach, we only use the properties of probability density function, especially the convolution properties. In this approach, we do not use any geometric properties of hyperdimensional space and this may be an advantage of this approach. The approach introduced in this short note may be helpful for the researchers who are interested in unsolved problems in the research area of hyperdimensional space, such as hyperpacking problem and hypercovering in information theory. 


\section{REFERENCES}

1. Collins G. P. The shapes of space. - Sci. Amer., 2004, v. 291, № 1, p. 94-103.

2. Конвей Дж., Слоэн Н. Упаковки шаров, решетки и группы. М.: Мир, 1990, 791 с.

3. Hamming R.W. Coding and Information Theory. Englewood Cliffs: Prentice-Hall, 1980, 239 p.

4. Papoulis A. Probabilty, Random Variables, and Stochastic Processes. New York: McGraw-Hill, 2002.

Поступила в редакцию

7.VII.2006

Исправленный вариант

22.VIII. 2008

(c) $2009 \Gamma$

PANG T.-X.* , LIN Z.-Y.*

\section{ON THE RATES OF THE CHUNG-TYPE LAW OF LOGARITHM ${ }^{1)}$}

Пусть $\left\{X, X_{n} ; n \geqslant 1\right\}$ - последовательность независимых одинаково распределенных случайных величин. Положим $S_{n}=X_{1}+X_{2}+\cdots+X_{n}$, $M_{n}=\max _{k \leqslant n}\left|S_{k}\right|, n \geqslant 1$. Используя метод сильной аппроксимации, мы получаем, что если $\mathbf{E} X=0, \mathbf{E} X^{2}=\sigma^{2}<\infty$ и $\mathbf{E} X^{2}(\ln |X|)^{b+1}<\infty$ для некоторого фиксированного $b>-1$, то

$$
\begin{aligned}
\lim _{\varepsilon \nearrow \infty} \varepsilon^{-2(b+1)} \sum_{n=1}^{\infty} \frac{(\ln n)^{b}}{n} \mathbf{P}\left(M_{n} \leqslant \varepsilon \sigma \sqrt{\frac{\pi^{2} n}{8 \ln n}}\right) \\
=\frac{4}{\pi} \Gamma(b+1) \sum_{k=0}^{\infty} \frac{(-1)^{k}}{(2 k+1)^{2 b+3}} .
\end{aligned}
$$

Обсуждается также сходимость моментов, сходимость в $L_{2}$ и сходимость почти наверное.

Ключевые слова и фразы: аппроксимация, сходимость моментов, сходимость в $L_{2}$, сходимость почти наверное, независимые одинаково распределенные случайные величины.

1. Introduction and main results. Let $\left\{X, X_{n} ; n \geqslant 1\right\}$ be a sequence of i.i.d. random variables with common distribution function $F$, mean 0 , and positive finite variance $\sigma^{2}$. Set $S_{n}=\sum_{k=1}^{n} X_{k}, M_{n}=\max _{k \leqslant n}\left|S_{k}\right|, n \geqslant 1$, and let $\ln n=\ln (n \vee e)$. Hsu and Robbins [11] and Erdős [7] established the well-known complete convergence

$$
\sum_{n=1}^{\infty} \mathbf{P}\left(\left|S_{n}\right| \geqslant \varepsilon n\right)<\infty, \quad \varepsilon>0
$$

* Department of Mathematics, Zhejiang University, Hangzhou 310027, China; e-mail: pangtianxiao@126.com; zlin@zju.edu.cn

1) Project supported by NSFC (No. 10871177, No. 10826034, and No. 10671176), Zhejiang Provincial Natural Science Foundation of China (No. R6090034), and Department of Education of Zhejiang Province (Y200803684). 